\title{
INDEX TO VOLUME 83
}

\section{INVITED ADDRESSES}

Alperin, J. L., Finite groups viewed locally, 1271

Ball, B. J., 112

Bers, Lipman, Quasiconformal mappings, with applications to differential equations, function theory and topology, 1083

Carlitz, L., Functions and correspondences in a finite field, 139

. 112

Chen, Kuo-Tsai, Iterated path integrals, 831

Coifman, Ronald R. and Weiss, Guido, Extensions of Hardy spaces and their use in analysis, 569

Comfort, W. W., Ultrafilters: Some old and some new results, 417 - 115

Conner, P. E. and Raymond, Frank, Deforming homotopy equivalences to homeomorphisms in aspherical manifolds, 36

Duren, Peter L., Coefficients of univalent functions, 891

- 107

Erdös, Paul, 113

Floyd, Edwin E., 107

Folland, G. B., Applications of analysis on nilpotent groups to partial differential equations, 912

Gardner, Robert B., New viewpoints in the geometry of submanifolds of $R^{N}, 1$

Gersten, Stephen M., 107

Goldschmidt, David M., 107

Hajian, Arshag B., 107

Helgason, Sigurdur, Invariant differential equations on homogeneous manifolds, 751

Hochster, Melvin, 107

Kalman, R., 112

Keen, Linda, A rough fundamental domain for Teichmüller spaces, 1199

Lacher, R. C., Cell-like mappings and their generalizations, 495

Lew, John S., 115

Liggett, Thomas M., 118

MacPherson, Robert, 115

Martin, Donald A., 107

May, J. P., Infinite loop space theory, 456

May, Peter, 107

Milgram, R. James, 118

Miller, Charles F. III, 107

Montgomery, Hugh L., 114

Nelson, Edward, Internal set theory: A new approach to nonstandard analysis, 1165

Noll, Walter, 107

Phelps, R. R., The Choquet representation in the complex case, 299

Powers, Robert T., 117

Raymond, Frank. See Conner, P. E.

Sachs, R. K. and Wu, H., General relativity and cosmology, 1101

Shiffman, Bernard, Holomorphic curves in algebraic manifolds, 553

Singer, I. M., 107

Smith, Kennan T., Solmon, Donald C. and Wagner, Sheldon L., Practical and mathematical aspects of the problem of reconstructing objects from radiographs, 1227

Solmon, Donald C. See Smith, Kennan T.

Spitzer, Frank, Stochastic time evolution of one dimensional infinite particle systems, 880 107 
Stoll, Wilhelm, Aspects of value distribution theory in several conplex variables, 166

Veech, William A., Topological dynamics, 775

Wagner, Sheldon L. See Smith, Kennan T.

Wainger, Stephen, 114

Weiss, Guido. See Coifman, Ronald R.

Wightman, Arthur S., 107

Wu, H. See Sachs, R. K.

\section{BOOK REVIEWS}

Aczél, J. See Kampé de Fériet, J.

Adams, J. F. A geometric approach to homology theory by S. Buoncristiano, C. P. Rourke and B. J. Sanderson, 222

Arbib, Michael A., Fuzzy sets and their applications to cognitive and decision processes edited by L. A. Zadeh, K.-S. Fu, K. Tanaka and M. Shimura; Introduction to the theory of fuzzy subsets. Volume I by A. Kaufmann; and Applications of fuzzy sets to systems analysis by C. V. Negoiţă and D. A. Ralescu, 946

Bair, Jacques. See Day, M. M.

Barnes, Donald W. See Barwise, Jon

Barwise, Jon. An algebraic introduction to mathematical logic by Donald W. Barnes and John M. Mack; An outline of mathematical logic by Andrzej Grzegorczyk; Completeness, compactness, and undecidability by Alfred B. Manaster; and Mathematical logic by Daniel Ponasse, 186

Beals, Richard. Basic linear partial differential equations by François Treves, 208

Benedetto, John J. See Ross, Kenneth A.

Bishop, Errett. Elementary calculus by H. Jerome Keisler, 205

Bogolubov, N. N. See Jaffe, Arthur

$$
\text { See Moser, Jürgen }
$$

Bondy, J. A. See Descartes, Blanche

Borovkov, A. A. See Kingman, J. F. C.

Bredon, Glen E. Cohomology theory of topological transformation groups by Wu Yi Hsiang, 711

Broughan, Kevin A. See Isbell, J. R.

Browder, Felix E. See Kaplansky, Irving

Brown, James R. See Jacobs, K.

Buoncristiano, S. See Adams, J. F.

Carrier, George F. See Payne, L. E.

Cherlin, Greg. See Eklof, Paul C.

Chern, S. S. Integral geometry and geometric probability by Luis A. Santaló, 1289

Conolly, Brian. See Neuts, Marcel F.

Cornea, Aurel. See Meyer, P. A.

Cozzens, John. See Goodearl, K. R.

Craig, William. See Diagneault, Aubert

Cuppens, Roger. See Helms, L. L.

Curtis, Charles W. Character theory of finite groups by I. Martin Isaacs, 1005

Daigneault, Aubert. Logic in algebraic form by William Craig; and An algebraic approach to non-classical logics by Helena Rasiowa, 189

Daróczy, Z. See Kampé de Fériet, J.

Davis, Martin. Mathematical logic by J. Donald Monk, 1007

Day, M. M. Etude géométrique des espaces vectoriels, une introduction by Jacques Bair and René Fourneau, 951

Denker, Manfred. See Jacobs, K.

Descartes, Blanche. Graph theory with applications by J. A. Bondy and U. S. R. Murty, 313 Dickmann, M. A. See Hanf, William P.

Drasin, David. Entire holomorphic mappings in one and several complex variables by Phillip A. Griffiths, 942

Dunkl, Charles F. See Hofmann, Karl H.

Eaton, Morris L. Techniques of multivariate calculation by Roger H. Farrell, 939

Edelstein, M. Embeddings and extensions in analysis by J. H. Wells and L. R. Williams, 220 
Eklof, Paul C. Model theoretic algebra: Selected topics by Greg Cherlin, 954

Elliott, Robert J. Pursuit games by Otomar Hájek, 243

Faith, Carl. See Goodearl, K. R.

Farrell, Roger H. See Eaton, Morris L.

Feferman, Solomon. Proof theory by Gaisi Takeuti, 351

Fefferman, Robert. Differentiation of integrals in $R^{n}$ by Miguel de Guzmán, 196

Fine, Terrence L. A mathematical theory of evidence by Glenn Shafer, 667

de Finetti, Bruno. See Good, I. J.

Fourneau, René. See Day, M. M.

Fu, K.-S. See Arbib, Michael A.

Fuller, Wayne A. See Hannan, E. J.

Galambos, János. See Volkmann, Bodo

Gastwirth, Joseph L. Nonparametrics: Statistical methods based on ranks by E. L. Lehmann, 967

Gilbert, William J. See Herstein, I. N.

Good, A. Applications of sieve methods to the theory of numbers by C. Hooley, 1015

Abstract analytic number theory by J. Knopfmacher, 1021

Good, I. J. Theory of probability, Volume I, II by Bruno de Finetti, 94

Goodearl, K. R. Simple Noetherian rings by John Cozzens and Carl Faith, 211

Grenander, U. See Leob, A. L.

Greub, W. See Samelson, H.,

Griffiths, Phillip A. See Drasin, David

Grzegorczyk, Andrzej. See Barwise, Jon

Gustafson, W. H. Matrix groups by D. A. Suprunenko, 318

de Guzmán, Miguel. See Fefferman, Robert

Hájek, Otomar. See Elliott, Robert J.

Halperin, Stephen. See Samelson, H.

Hanf, William P. Large infinitary languages, model theory by M. A. Dickmann, 184

Hannan, E. J. Introduction to statistical time series by Wayne A. Fuller, 989

Helms, L. L. Decomposition of multivariate probabilities by Roger Cuppens, 699

Herstein, I. N. Modern algebra with applications by William J. Gilbert, 1286

Hestenes, Magnus R. See Jeroslow, Robert G.

Heyting, A. See Kreisel, G.

Hille, Einar. See Sibuya, Yasutaka

Hirsch, Morris W. See James, I. M.

Hofmann, Karl H. Representations of commutative semitopological semigroups by Charles $\mathrm{F}$. Dunkl and Donald E. Ramirez, 236

Hoobler, R. T. Sheaf theory by B. R. Tennison, 702

Hooley, C. See Good, A.

Hsiang, Wu Yi. See Bredon, Glen E.

Humphreys, J. E. Rational methods in Lie algebras by George B. Seligman, 993

Hyers, D. H. The theory of approximate methods and their application to the numerical solu. tion of singular integral equations by V. V. Ivanov, 964

Isaacs, I. Martin. See Curtis, Charles W.

Isbell, J. R. Invariants for real-generated uniform topological and algebraic categories by Kevin A. Broughan, 200

Ivanov, V. V. See Hyers, D. H.

Jacobs, K. Ergodic theory on compact spaces by Manfred Denker and Ergodic theory and topological dynamics by James R. Brown, 1294

Jaffe, Arthur. Introduction to axiomatic quantum field theory by N. N. Bogolubov, A A. Logunov, and I. T. Todorov, 349

James, I. M. Differential topology by Morris W. Hirsch, 997

Jenkins, James A. Univalent functions-selected topics by Glenn Schober, 215

Jeroslow, Robert G. Optimization theory, the finite dimensional case by Magnus R. Hestenes, 324

Johnson, D. L. See Rotman, Joseph

Jones, D. S. Asymptotic wave theory by Maurice Roseau, 315 
Kampé de Fériet, J. On measures of information and their characterizations by J. Aczél and

Z. Daróczy, 192

Kaplansky, Irving. Mathematical developments arising from Hilbert problems edited by Felix

E. Browder, 697

Karpovsky, M. G. See Stone, Harold S.

Kaufmann, A. See Arbib, Michael A.

Kazarinoff, Nicholas D. The Hopf bifurcation and its applications by J. E. Marsden and M. McCracken, 998

Keesling, James. Dimension theory of general spaces by A. R. Pears, 203

Keisler, H. Jerome. See Bishop, Errett.

Keown, R. Unitary representations and harmonic analysis, an introduction by Mitsuo Sugiura, 100

Kesten, Harry. Sums of independent random variables by V. V. Petrov, 696

Kingman, J. F. C. Stochastic processes in queueing theory by A. A. Borovkov, 317

Kluvanek, Igor. See Uhl, J. J., Jr.

Knopfmacher, J. See Good, A.

Knowles, Greg. See Uhl, J. J., Jr.

Kreisel, G. L. E. J. Brouwer collected works, Volume I, Philosophy and foundations of mathematics edited by A. Heyting, 86

Lakshmikantham, V. Introduction to spectral theory: Selfadjoint ordinary differential operators by B. M. Levitan and I. S. Sargsjan, 188

Lehmann, E. L. See Gastwirth, Joseph L.

Leob, A. L. Pattern synthesis, lectures in pattern recognition, Volume 1 by $\mathrm{U}$. Grenander, 958

Levitan, B. M. See Lakshmikantham, V.

Licea, Gabriela. See Meyer, P. A.

Lightstone, A. H. See Loeb, Peter A.

Loeb, Peter A. Non-archimedean fields and asymptotic expansions by A. H. Lightstone and

A. Robinson, 231

Logunov, A. A. See Jaffe, Arthur

Mack, John M. See Barwise, Jon

Malita, Mircea. See Snell, Laurie

Manaster, Alfred B. See Barwise, Jon

Manes, E. G. Topological transformation Groups 1: A categorical approach by J. de Vries, 720

Marsden, J. E. See Kazarinoff, Nicholas D.

Mazur, Barry. Ernst Edward Kummer, Collected Papers, Vols. I and II edited by André Weil, 976

McCracken, M. See Kazarinoff, Nicholas D.

Meyer, P. A. Order and potential resolvent families of kernels by Aurel Cornea and Gabriela Licea, 672

Mitropoliskii, Ju. A. See Moser, Jürgen

Monk, J. Donald. See Davis, Martin

Morse, Marston. See Smale, Stephen

Moser, Jürgen. Methods of accelerated convergence in nonlinear mechanics by N. N.

Bogoljubov, Ju. A. Mitropoliskii and A. M. Samoilenko, 1290

Murty, U. S. R. See Descartes, Blanche

Negoiţă, C. V. See Arbib, Michael A.

Neuts, Marcel F. Lecture notes on queueing systems by Brian Conolly, 322

Neuwirth, Lee P. Knots and links by Dale Rolfsen, 931

Nikol'skir, S. M. See Taibleson, M. H.

Payne, L. E. Partial differential equations: theory and technique by Geroge F. Carrier and Carl E. Pearson, 718

Pears, A. R. See Keesling, James

Pearson, Carl E. See Payne, L. E.

Petrov, V. V. See Kesten, Harry

Piron, C. See Varadarajan, V. S. 
Ponasse, Daniel. See Barwise, Jon

Ralescu, D. A. See Arbib, Michael A.

Ramirez, Donald E. See Hofmann, Karl H.

Rasiowa, Helena. See Daigneault, Aubert

Robinson, A. See Loeb, Peter A.

Rolfsen, Dale. See Neuwirth, Leo P.

Roseau, Maurice. See Jones, D. S.

Ross, Kenneth A. Spectral synthesis by John J. Benedetto, 960

Rotman, Joseph. Presentation of groups by D. L. Johnson, 97

Rourke, C. P. See Adams, J. F.

Samelson, H. Connections, curvature, and cohomology. Vols. I, II, III by W. Greub, Stephen Halperin and Ray Vanstone, 1011

Samoilenko, A. M. See Moser, Jürgen

Sanderson, B. J. See Adams, J. F.

Santaló, Luis A. See Chern, S. S.

Sargsjan, I. S. See Lakshmikantham, V.

Schober, Glenn. See Jenkins, James A.

Segal, Irving Ezra. See Taub, A. H.

Seligman, George B. See Humphreys, J. E.

Shafer, Glenn. See Fine, Terrence $L$.

Shimura, M. See Arbib, Michael A.

Sibuya, Yasutaka. Ordinary differential equations in the complex domain by Einar Hille, 935

Smale, Stephen. Global variational analysis: Weierstrass integrals on a Riemannian manifold by Marston Morse, 683

Small, Lance W. Rings of quotients by Bo Stenström, 693

Snell, Laurie. Mathematics of organization by Mircea Malita and Corneliu Zidăroiu, 676

Stenström, Bo. See Small, Lance W.

Stone, Harold S. Finite orthogonal series in the design of digital devices by M. G. Karpovsky, 681

Sugiura, Mitsuo. See Keown, R.

Suprunenko, D. A. See Gustafson, W. H.

Taibleson, M. H. Approximation of functions of several variables and imbedding theorems, by $\mathrm{S}$. M. Nikol'skiI, 335

Takeuti, Gaisi. See Feferman, Solomon

Tanaka, K. See Arbib, Michael A.

Taub, A. H. Mathematical cosmology and extragalactic astronomy by Irving Ezra Segal, 705

Tennison, B. R. See Hoobler, R. T.

Todorov, I. T. See Jaffe, Arthur

Treves, François. See Beals, Richard

Ueno, Kenji. See Winters, Gayn B.

Uhl, J. J., Jr. Vector measures and control systems by Igor Kluvanek and Greg Knowles, 103

Vanstone, Ray. See Samelson, $\mathrm{H}$.

Varadarajan, V. S. Foundations of quantum physics by C. Piron, 226

Volkmann, Bodo. Representations of real numbers by infinite series by János Galambos, 973 de Vries, J. See Manes, E. G.

Weil, André. See Mazur, Barry

Wells, J. H. See Edelstein, M.

Williams, L. R. See Edelstein, M.

Winters, Gayn B. Classification theory of algebraic varieties and compact complex spaces by Kenji Ueno, 344

Zadeh, L. A. See Arbib, Michael A.

Zidăroiu, Corneliu. See Snell, Laurie

\section{RESEARCH ANNOUNCEMENTS}

Akbalut, Selman and King, Henry C. Real algebraic variety structures on P. L. manifolds, 281 Aldous, David J. Subsequences of sequences of random variables, 121

Alspach, Dale E. Quotients of $C[0,1]$ with separable dual, 1057 
Amitsur, S. A. and Small, Lance W. Prime PI-rings, 249

Arthur, James. A truncation process for reductive groups, 748

Ash, Avner. Cohomology of subgroups of finite index of $\operatorname{SL}(3, \mathrm{Z})$ and $\operatorname{SL}(4, \mathrm{Z}), 367$

Baker, Daniel. On a class of foliations and the evaluation of their characteristic classes, 394

Barrow, D. L., Chui, C. K., Smith, P. W. and Ward, J. D. Unicity of best $L_{2}$ approximation by second-order splines with variable knots, 1049

Baouendi, M. S. and Zachmanoglou, E. C. Unique continuation theorems for solutions of partial differential equations, 1045

Bennett, Mary Katherine. Every finite lattice is a face lattice, 406

Berger, M. S. and Plastock, R. A. On the singularities of nonlinear Fredholm operators, 1316

Berman, Abraham and Ward, Robert C. Stability and semipositivity of real matrices, 262

Böhme, R. and Tromba, A. J. The number of solutions to the classical Plateau problem is generically finite, 1043

Bottkol, M. Bifurcation of periodic orbits on manifolds, and Hamiltonian systems, 1060

Byrd, Richard D., Lloyd, Justin T., Pedersen, Franklin D. and Stepp James W. Automorphisms of semigroups of complexes of Abelian groups, 260

Chen, C.-S. Tight and taut embeddings and projective transformation, 1066

Chen, Su-shing. Nonpositively curved manifolds, 119

Chui, C. K. See Barrow, D. L.

Conze, J. P. and Ngoc, N. Dang. Noncommutative ergodic theorems, 1297

Cowen, M. J. and Douglas, R. G. Complex geometry and operator theory, 131

Cowling, Michael. The Kunze-Stein phenomenon, 293

Dales, H. G. and Esterle, J. Discontinuous homomorphisms from $C(X), 257$

Douglas, R. G. See Cowen, M. J.

Esterle, J. See Dales, H. G.

Farrell, F. T. and Jones, L. E. Markov cell structures, 739

Feshbach, Mark. The transfer and compact Lie groups, 372

Friedlander, Eric M. and Seymour, R. M. Two proofs of the stable Adams conjecture, 1300

Friedman, S. D. and Sacks, G. E. Inadmissable recursion theory, 255

Gerth, Frank III. The Hasse norm principle for Abelian extensions of number fields, 264

Giffen, Charles H. Hermitian forms and higher algebraic $K$-theory, 1303

Gross, Kenneth I., Holman, Wayne J. III and Kunze, Ray A. The generalized gamma function, new Hardy spaces, and representations of holomorphic type for the conformal group, 412

Hanscombe, K. P. Classification of involutive Banach-Lie algebras, 1063

Heitsch, James L. Residues and characteristic classes of foliation, 397

Hendriks, Harrie. Obstruction theory in 3-dimensional topology: classification theorems, 737

Herb, R. A. and Sally, P. J., Jr. Singular invariant eigendistributions as characters, 252

Holman, Wayne J. III. See Gross, Kenneth I.

Holton, D. A. See Little, C. H. C.

Jones, L. See Farrell, F. T.

Jones, William B. and Thron, W. J. Two-point Padé tables and T-fractions, 388

Kane, Richard. Torsion in the homology of H-spaces, 1041

Karp, Leon. Parallel vector fields and the topology of manifolds, 1051

Kilgore, T. A. Optimization of the norm of the Lagrange interpolation operator, 1069

King, Henry C. See Akbalut, Selman

Krengel, Ulrich and Sucheston, Louis. Semiamarts and finite values, 745

Kunze, Ray A. See Gross, Kenneth 1.

Lang, S. and Trotter, H. Primitive points on elliptic curves, 289

Leon, Jeffrey S. and Sims, Charles C. The existence and uniqueness of a simple group genera. ted by $\{3,4\}$-transpositions, 1039

Little, C. H. C. and Holton, D. A. A new characterization of planar graphs, 137

Lloyd, Justin T. See Byrd, Richard D.

Mandelbaum, R. Algebraic surfaces and irrational connected sums of four manifolds, 369

Meeks, William $\mathrm{H}_{3}$. III. The conformal structure and geometry of triply periodic minimal sur. faces in $\mathbf{R}^{3}, 134$

- Representing homology classes by embedded circles and the existence of circles invariant under isometries, 1037 
Megiddo, Nimrod. A good algorithm for lexicographically optimal flows in multi-terminal networks, 407

Menzala, G. Perla. Inverse scattering for the Klein-Gordon equation, 735

Micchelli, C. A. and Pinkus, A. Best mean approximation to a 2-dimensional kernel by tensor products, 400

Milnor, Tilla Klotz. A characterization of harmonic immersions of surfaces, 403

Moishezon, B. Some estimations in the topology of simply-connected algebraic surfaces, 741

Ně̌etril, Jaroslav and Rödl, Vojtěch. A structural generalization of the Ramsey theorem, 127

Ngoc, N. Dang. See Conze, J. P.

Pedersen, Franklin D. See Byrd, Richard D.

Pichorides, S. K. A remark on exponential sums, 283

Pigno, Louis. Parts of measures of integer-valued transforms, 279

Pinkus, A. See Micchelli, C. A.

Pitt, Loren D. Closure theorems for spaces of entire functions, 732

Plastock, R.A. See Berger, M. S.

Pryde, A. J. Second order elliptic equations with mixed boundary conditions, 391

Rallis, S. and Schiffmann, G. Discrete spectrum of the Weil representation, 267

- Automorphic cusp forms constructed from the Weil representation, 271

Rödl, Vojtěch. See Nešetril, Jaroslav.

Rowen, Louis Halle. Central simple algebras with involution, 1031

Sacks, G. E. See Friedman, S. D.

Sacks, J. and Uhlenbeck, K. The existence of minimal immersions of two-spheres, 1033

Saff, E. B. and Varga, R. S. Nonuniqueness of best approximating complex rational functions, 375

Sally, P. J., Jr. See Herb, R. A.

Schechter, Martin. The existence of wave operators in scattering theory, 381

Schelter, W. Affine PI rings are catenary, 1309

Schiffmann, G. See Rallis, S.

Schwarz, Gerald W. Covering smooth homotopies of orbit spaces, 1028

Seshardi, C. S. Moduli of vector bundles on curves with parabolic structures, 124

Seymour, R. M. See Friedlander, Eric M.

Sherman, Thomas O. Fourier analysis on compact symmetric space, 378

Shyr, Jih-Min. Class numbers of totally positive binary forms over totally real numberfields, 286

Sibuya, Yasutaka. Stokes phenomena, 1075

Sims, Charles C. See Leon, Jeffrey S.

Singer, Michael. Determination of the augmentation terminal for finite abelian groups, 1321

Small, Lance W. See Amitsur, S. A.

Smeltzer, Michael D. Subadditive stochastic processes, 1054

Smith, P. W. See Barrow, D. L.

Snaith, Victor. Towards algebraic cobordism, 384

Sourour, A. R. On the isometries of $L^{p}(\Omega X), 129$

Stark, H. M. Hilbert's twelfth problem and L-series, 1072

Stepp, James W. See Byrd, Richard D.

Sucheston, Louis. See Krengel, Ulrich

Sussmann, Héctor J. An interpretation of stochastic differential equations as ordinary differential equations which depend on the sample point, 296

Thron, W. J. See Jones, William B.

Tolimieri, R. The multiplicity problem for 4-dimensional solvmanifolds, 365

Tromba, A. J. See Böhme, R.

Trotter, H. See Lang, S.

Tzimbalario, J. Derivatives of polynomials of best approximation, 1311

Uhlenbeck, K. See Sacks, J.

Varga, R. S. See Saff, E. B.

Venema, Gerard A. A topological disk in a 4-manifold can be approximated by plecewise linear disks, 386

Ward, J. D. See Barrow, D. L.

Ward, Robert C. See Berman, Abraham 
Wells, G. S. Spaces of smooth functions on analytic sets, 276

Winters, Gayn B. Obstructed one dimensional local complete intersections, 410

Wolpert, Scott. The eigenvalue spectrum as moduli for compact Riemann surfaces, 1306

Yau, Stephen Shing-Toung. On almost minimally elliptic singularities, 362

The signature of smoothings of higher dimensional singularities, 1313

Zachmanoglou, E. C. See Baouendi, M. S.

Zafran, Misha. The functions operating on homogeneous Banach algebras, 1319

Zimmer, Robert J. Amenable ergodic actions, hyperfinite factors and Poincaré flows, 1078

\section{MISCELLANEOUS}

Index, 1323

Meetings,

The January meeting in San Antonio, 107

The March meeting in Tallahassee, 112

The March meeting in Urbana, 113

The April meeting in New York, 115

The April meeting in Reno, 117

The June meeting in Portland, 118

Macintyre, Angus J. Obituary of Abraham Robinson, 1918-1974, 646

Wilder, R. L. Erratum, Volume 82, 415 